\title{
New approach on differential equation via trapezoidal neutrosophic number
}

\author{
I. R. Sumathi ${ }^{1}$ - C. Antony Crispin Sweety ${ }^{2}$
}

Received: 23 April 2019 / Accepted: 2 August 2019 / Published online: 16 August 2019

(c) The Author(s) 2019

\begin{abstract}
Neutrosophic Logic is a tool based on non-standard analysis to represent mathematical model of uncertainty, vagueness, ambiguity, incompleteness, and inconsistency. In Neutrosophic set, indeterminacy is quantified explicitly whereas the truth membership, indeterminacy membership, and falsity membership are independent. This plays a vital role in many situations when we handle inconsistent and incomplete information. In modeling problems, differential equations have major applications in the field of science and engineering and the study of differential equation with uncertainty is one of emerging field of research. In this paper, the differential equations in neutrosophic environment are explored, also the solution of second-order linear differential equation with trapezoidal neutrosophic numbers as boundary conditions is discussed. Furthermore, the numerical example is given to demonstrate the solution with different values of $(\alpha, \beta, \gamma)$-cut of trapezoidal neutrosophic number.
\end{abstract}

Keywords Neutrosophic set · Trapezoidal neutrosophic number $\cdot$ Neutrosophic differential equation

Mathematics Subject Classification 03E72

\section{Introduction}

Neutrosophic set is the generalization of classical set, fuzzy set [1], intuitionistic fuzzy set [2,3], and so on which highlights the origin and nature of neutralities in different fields. This multifaceted logic was introduced by $\mathrm{F}$. Smarandache [4-6] which imports the term indeterminacy and carries more information than fuzzy logic. This leads to give the better performance than fuzzy logic. In neutrosophic logic, a proposition has a degree of truth $(T)$, a degree of indeterminacy $(I)$, and a degree of falsity $(F)$, where $T, I$, and $F$ are standard or non-standard subsets of $]-0,1+[$. However it is difficult to handle data with non-standard interval, and hence, the [7] single-valued neutrosophic set was introduced which takes the values in the standard interval $[0,1]$.

$凶$ I. R. Sumathi

sumathi_raman2005@yahoo.co.in

C. Antony Crispin Sweety

antonycrispinsweety@gmail.com

1 Amrita School of Engineering, Amrita Vishwa

Vidyapeetham, Coimbatore, India

2 Nirmala College for Women, Coimbatore, India
The notion of neutrosophic measure, neutrosophic integral, and neutrosophic probability were introduced by Smarandache [8]. Many practical examples are presented in neutrosophic measure, and consequently, the neutrosophic integral and neutrosophic probability are also defined in many ways, because there are various types of indeterminacies, depending on the problem. Many researchers have applied the neutrosophic logic in various fields.

Single-valued neutrosophic numbers, triangular neutrosophic numbers and trapezoidal neutrosophic numbers, and their application in decision-making are explored in [9-11]. The neutrosophic number from different view points are introduced [12] and the different types of linear and nonlinear generalized triangular neutrosophic numbers which are very important for uncertainty theory and de-neutrosophication concept for neutrosophic number for triangular neutrosophic numbers are discussed that helps to convert a neutrosophic number into a crisp number. This has been applied in imprecise project evaluation review technique and route selection problem. Abdel-Basset et al. [13] introduced a advanced type of neutrosophic technique, called type 2 neutrosophic numbers(T2NN), and a real case dealing with a decision-making problem based on T2NN-TOPSIS (Technique for order preference by similarity to ideal solution) 
methodology to prove the efficiency and the applicability of the type 2 neutrosophic number were illustrated. The triangular neutrosophic numbers (TriNs) were used to present the linguistic variables based on opinions of experts and decision-makers. The problem of supplier selection in sustainable supplier chain management (SSCM) is also incorporated in [14]. The multicriteria decision-making (MCDM) methodology is one of the keys for solving complicated and complex decision problems. In [15], bipolar neutrosophic number was defined and Group Decision-Making based on Neutrosophic TOPSIS approach has been applied for Smart Medical Device Selection. Neutrosophic logic helps in preventing the loss of data, and hence, it has been applied in many decision-making problems [16-20].

The Internet of Things (IoT) is the network of physical devices and the network connectivity enables these objects to collect and exchange data. The IoT allows objects to be sensed or controlled remotely across existing network infrastructure, creating opportunities for more direct integration of the physical world into computer-based systems, and resulting in improved efficiency, accuracy, and economic benefit in addition to reduced human intervention. The IoT has the potential to add a new dimension by enabling communications with and among smart objects, thus leading to the vision of "anytime, anywhere, anymedia, anything" communications. IoT is one of the challenging and emerging fields of research in science and engineering. Nabeeh et al. [21] presented a neutrosophic analytical hierarchy process (AHP) of the IoT in enterprises to help decision-makers to estimate the influential factors.

Differentiation plays an important role in the field of science and engineering. Many problems arise with uncertain or imprecise parameters. To model this uncertainty, we develop the differential equation with imprecise parameters. Fuzzy differential equation [22-30] has been introduced to model this uncertainty. However, it considers only the membership value. Later, intuitionistic fuzzy differential equation [31-36] was emerged with degree of membership and non-membership. However, these two logic does not have the term indeterminacy. Hence, neutrosophic differential equation was developed to model the indeterminacy. Smarandache [37] initiated the concept of neutrosophic function such as exponential function, neutrosophic logarithmic function, and neutrosophic inverse function. Also he introduced, neutrosophic calculus, which studies the neutrosophic limits, neutrosophic derivatives, and neutrosophic integrals. Differential equation with uncertainty in a growing area.The differential equations with neutrosophic numbers is studied in [38]. The multifaceted factors of neutrosophic numbers have been exemplified in higher order differential equation. The structure of the paper is organized as follows. In Preliminary section, the pre-requisite concepts are given and the conditions for strong solution are defined for solving the second-order differential equations. Followed by preliminaries, the solution of second-order differential equation with trapezoidal neutrosophic number as boundary condition is derived. Finally, the numerical example is illustrated and its graphical interpretations are also shown. In the conclusion part, the future research scope is discussed.

\section{Preliminaries}

Definition 1 [4] Let $X$ be a universe set. A neutrosophic set $A$ on $X$ is defined as $A=\left\{\left\langle T_{A}(x), I_{A}(x), F_{A}(x)\right\rangle: x \in X\right\}$, where $\left.T_{A}(x), I_{A}(x), F_{A}(x): X \rightarrow^{-}\right] 0,1\left[^{+}\right.$represents the degree of membership, degree of indeterministic, and degree of non-membership respectively of the element $\mathrm{x} \in X$, such that ${ }^{-} 0 \leq T_{A}(x)+I_{A}(x)+F_{A}(x) \leq 3^{+}$.

Definition 2 [7] Let $X$ be a universe set. A single-valued neutrosophic set $A$ on $X$ is defined as $A=\left\{\left\langle T_{A}(x), I_{A}(x), F_{A}(x)\right\rangle\right.$ $: x \in X\}$, where $T_{A}(x), I_{A}(x), F_{A}(x): X \rightarrow[0,1]$ represents the degree of membership, degree of indeterministic, and degree of non-membership, respectively, of the element $\mathrm{x} \in \mathrm{X}$, such that $0 \leq T_{A}(x)+I_{A}(x)+F_{A}(x) \leq 3$.

Definition $3(\alpha, \beta, \gamma)$-cut: The $(\alpha, \beta, \gamma)$-cut neutrosophic set is denoted by $F_{(\alpha, \beta, \gamma)}$, where $\alpha, \beta, \gamma \in[0,1$ and are fixed numbers, such that $\alpha+\beta+\gamma \leq 3$ is defined as $F_{\alpha, \beta, \gamma}=\left\{\left\langle T_{A}(x), I_{A}(x), F_{A}(x)\right\rangle: x \in X, T_{A}(x) \geq\right.$ $\left.\alpha, I_{A}(x) \leq \beta, F_{A}(x) \leq \gamma\right\}$.

Definition 4 A neutrosophic set $A$ defined on the universal set of real numbers $R$ is said to be neutrosophic number if it has the following properties.

(i) $A$ is normal if there exist $x_{0} \in R$, such that $T_{A}\left(x_{0}\right)=$ $1\left(I_{A}\left(x_{0}\right)=F_{A}\left(x_{0}\right)=0\right)$.

(ii) $\mathrm{A}$ is convex set for the truth function $T_{A}(x)$, i.e., $T_{A}\left(\mu x_{1}+(1-\mu) x_{2}\right) \geq \min \left(T_{A}\left(x_{1}\right), T_{A}\left(x_{2}\right)\right) \forall x_{1}, x_{2} \in$ $R, \mu \in[0,1]$.

(iii) $A$ is concave set for the indeterministic function and false function $I_{A}(x)$ and $F_{A}(x)$, i.e., $I_{A}\left(\mu x_{1}+(1-\mu) x_{2}\right) \geq \max \left(I_{A}\left(x_{1}\right), I_{A}\left(x_{2}\right)\right) \forall x_{1}, x_{2} \in$ $R, \mu \in[0,1]$.

$F_{A}\left(\mu x_{1}+(1-\mu) x_{2}\right) \geq \max \left(F_{A}\left(x_{1}\right), F_{A}\left(x_{2}\right)\right) \forall x_{1}, x_{2} \in$ $R, \mu \in[0,1]$.

Definition 5 [37] A trapezoidal neutrosophic number $A$ is a subset of neutrosophic number in $\mathrm{R}$ with the following truth function, indeterministic function, and falsity function which is given by the following: 


$$
\begin{aligned}
& T_{A}(x)=\left\{\begin{array}{cl}
\left(\frac{x-a}{b-a}\right) \vartheta_{A} & \text { for } a \leq x \leq b \\
\vartheta_{A} & \text { for } b \leq x \leq c \\
\left(\frac{d-x}{d-c}\right) \vartheta_{A} & \text { for } c \leq x \leq d \\
0 & \text { otherwise }
\end{array}\right. \\
& I_{A}(x)=\left\{\begin{array}{cl}
\left(\frac{b-x}{b-a}\right) v_{A} & \text { for } a \leq x \leq b \\
v_{A} & \text { for } b \leq x \leq c \\
\left(\frac{d-x}{d-c}\right) v_{A} & \text { for } c \leq x \leq d \\
1 & \text { otherwise }
\end{array}\right. \text { and } \\
& F_{A}(x)=\left\{\begin{array}{cl}
\left(\frac{b-x}{b-a}\right) \kappa_{A} & \text { for } a \leq x \leq b \\
\kappa_{A} & \text { for } b \leq x \leq c \\
\left(\frac{d-x}{d-c}\right) \kappa_{A} & \text { for } c \leq x \leq d \\
1 & \text { otherwise }
\end{array}\right.
\end{aligned}
$$

where $a \leq b \leq c \leq d$ and a trapezoidal neutrosophic number is denoted by $A_{T R N}=\left\langle(a, b, c, d) ; \vartheta_{A}, v_{A}, \kappa_{A}\right\rangle$.

Note 1: Here, $T_{A}(x)$ increases with constant rate for $x \in$ $[a, b]$ and decreases for $x \in[c, d]$, but $I_{A}(x)$ and $F_{A}(x)$ decreases with constant rate for $x \in[a, b]$ and increases for $x \in[c, d]$

Definition $6(\alpha, \beta, \gamma)$-cut of a trapezoidal neutrosophic number $A_{T R N}=\left\langle(a, b, c, d) ; \vartheta_{A}, v_{A}, \kappa_{A}\right\rangle$ is defined as follows: $A_{\alpha, \beta, \gamma}=\left[A_{1}(\alpha), A_{2}(\alpha)\right] ;\left[A_{1}^{\prime}(\beta), A_{2}^{\prime}(\beta)\right] ;\left[A_{1}^{\prime \prime}(\gamma), A_{2}^{\prime \prime}(\gamma)\right]$, $0 \leq \alpha+\beta+\gamma \leq 3$, where

$\left[A_{1}(\alpha), A_{2}(\alpha)\right]=\left[(a+\alpha(b-a)) \vartheta_{A},(d-\alpha(d-c)) \vartheta_{A}\right]$ $\left[A_{1}^{\prime}(\beta), A_{2}^{\prime}(\beta)\right]=\left[(b-\beta(b-a)) v_{A},(c+\beta(d-c)) v_{A}\right]$, $\left[A_{1}^{\prime \prime}(\gamma), A_{2}^{\prime \prime}(\gamma)\right]=\left[(b-\gamma(b-a)) \kappa_{A},(c+\gamma(d-c)) \kappa_{A}\right]$

Definition 7 [28] The derivative of a fuzzy valued function $f:(a, b) \rightarrow R$ at $x_{0}$ is defined as follows:

$f^{\prime}\left(x_{0}\right)=\lim _{h \rightarrow 0} \frac{f\left(x_{0}+h\right)-f\left(x_{0}\right)}{h}$ and

$f^{\prime}\left(x_{0}\right)$ is $\mathscr{D}^{1}$-differentiable at $x_{0}$ if

$\left[f^{\prime}\left(x_{0}\right)\right]_{\alpha}=\left[f_{1}^{\prime}\left(x_{0}, \alpha\right), f_{2}^{\prime}\left(x_{0}, \alpha\right)\right]$ and

$f^{\prime}\left(x_{0}\right)$ is $\mathscr{D}^{2}$-differentiable at $x_{0}$ if

$\left[f^{\prime}\left(x_{0}\right)\right]_{\alpha}=\left[f_{2}^{\prime}\left(x_{0}, \alpha\right), f_{1}^{\prime}\left(x_{0}, \alpha\right)\right]$

for all $\alpha \in[0,1]$.
Definition 8 [27] The second-order derivative of a fuzzy valued function $f:(a, b) \rightarrow R$ at $x_{0}$ is defined as follows:

$f^{\prime \prime}\left(x_{0}\right)=\lim _{h \rightarrow 0} \frac{f^{\prime}\left(x_{0}+h\right)-f^{\prime}\left(x_{0}\right)}{h}$ and

$f^{\prime}\left(x_{0}\right)$ is $\mathscr{D}^{1}$-differentiable at $x_{0}$ if

$f^{\prime \prime}\left(x_{0}, \alpha\right)=\left\{\begin{array}{c}\left(f_{1}^{\prime}\left(x_{0}, \alpha\right), f_{2}^{\prime}\left(x_{0}, \alpha\right)\right) \text { if } f \text { is } \\ D^{1} \text {-differentiable on }(a, b) \\ \left(f_{2}^{\prime}\left(x_{0}, \alpha\right), f_{1}^{\prime}\left(x_{0}, \alpha\right)\right) \text { if } f \text { is } \\ D^{2} \text {-differentiable on }(a, b)\end{array}\right.$

for all $\alpha \in[0,1]$ and $f^{\prime}\left(x_{0}\right)$ is $\mathscr{D}^{2}$-differentiable at $x_{0}$ if

$f^{\prime \prime}\left(x_{0}, \alpha\right)=\left\{\begin{array}{c}\left(f_{2}^{\prime}\left(x_{0}, \alpha\right), f_{1}^{\prime}\left(x_{0}, \alpha\right)\right) \text { if } f \text { is } \\ D^{1} \text {-differentiable on }(a, b) \\ \left(f_{1}^{\prime}\left(x_{0}, \alpha\right), f_{2}^{\prime}\left(x_{0}, \alpha\right)\right) \text { if } f \text { is } \\ D^{2} \text {-differentiable on }(a, b)\end{array}\right.$

for all $\alpha \in[0,1]$.

Definition 9 Let the solution of the neutrosophic differential equation be $y(x)$ and its $(\alpha, \beta, \gamma)$-cut be $[y(x, \alpha$, $\beta, \gamma)=\left[\left(y_{1}(x, \alpha), y_{2}(x, \alpha),\left(y_{1}^{\prime}(x, \beta), y_{2}^{\prime}(x, \beta),\left(y_{1}^{\prime \prime}(x, \gamma)\right.\right.\right.\right.$, $\left.y_{2}^{\prime \prime}(x, \gamma)\right]$ The solution is a strong solution if

(i) $\frac{\mathrm{d} y_{1}(x, \alpha)}{\mathrm{d} \alpha}>0, \frac{\mathrm{d} y_{2}(x, \alpha)}{\mathrm{d} \alpha}<0 \forall \alpha \in[0,1], y_{1}(x, 1) \leq y_{2}(x, 1)$.

(ii) $\frac{\mathrm{d} y_{1}^{\prime}(x, \beta)}{\mathrm{d} \beta}<0, \frac{\mathrm{d} y_{2}^{\prime}(x, \beta)}{\mathrm{d} \beta}>0 \forall \beta \in[0,1], y_{1}^{\prime}(x, 0) \leq y_{2}^{\prime}(x, 0)$.

(iii) $\frac{\mathrm{d} y_{1}^{\prime \prime}(x, \gamma)}{\mathrm{d} \gamma}<0, \frac{\mathrm{d} y_{2}^{\prime \prime}(x, \gamma)}{\mathrm{d} \gamma}>0 \forall \gamma \in[0,1], y_{1}^{\prime \prime}(x, 0) \leq y_{2}^{\prime \prime}(x, 0)$.

\section{Solution of second-order neutrosophic differential equation}

Consider the differential equation $\frac{\mathrm{d}_{2} y(x)}{\mathrm{d} x_{2}}=p y(x)$ with the boundary condition $y(0)=\tilde{u}$ and $y(l)=\tilde{v}$, where $\tilde{u}, \tilde{v}$ are trapezoidal neutrosophic number.

Let $\tilde{u}=\left\{\left(u_{1}, u_{2}, u_{3}, u_{4}\right) ; \vartheta_{1}, v_{1}, \kappa_{1}\right\}$ and $\tilde{v}=\left\{\left(v_{1}, v_{2}, v_{3}\right.\right.$, $\left.\left.v_{4}\right) ; \vartheta_{2}, v_{2}, \kappa_{2}\right\}$.

1. When $p$ is positive constant, i.e., $p>0$. then two cases are possible.

Case 1: $y(x)$ and $\frac{\mathrm{d} y(x)}{\mathrm{d} x}$ are $\mathscr{D}^{1}$-differentiable or $\mathscr{D}^{2}$-differen tiable, and then, we have the following:

$$
\begin{array}{ll}
\frac{\mathrm{d}^{2} y_{1}(x, \alpha)}{\mathrm{d} x^{2}}=p y_{1}(x, \alpha) ; & \frac{\mathrm{d}^{2} y_{2}(x, \alpha)}{\mathrm{d} x^{2}}=p y_{2}(x, \alpha) \\
\frac{\mathrm{d}^{2} y_{1}^{\prime}(x, \beta)}{\mathrm{d} x^{2}}=p y_{1}^{\prime}(x, \beta) ; & \frac{\mathrm{d}^{2} y_{2}^{\prime}(x, \beta)}{\mathrm{d} x^{2}}=p y_{2}^{\prime}(x, \beta) \\
\frac{\mathrm{d}^{2} y_{1}^{\prime \prime}(x, \gamma)}{\mathrm{d} x^{2}}=p y_{1}^{\prime \prime}(x, \gamma) ; & \frac{\mathrm{d}^{2} y_{2}^{\prime \prime}(x, \gamma)}{\mathrm{d} x^{2}}=p y_{2}^{\prime \prime}(x, \gamma)
\end{array}
$$


with the boundary conditions:

$$
\begin{aligned}
& y_{1}(0, \alpha)=\left(u_{1}+\alpha\left(u_{2}-u_{1}\right)\right) \vartheta_{1}, \\
& y_{2}(0, \alpha)=\left(u_{4}-\alpha\left(u_{4}-u_{3}\right)\right) \vartheta_{1}, \\
& y_{1}^{\prime}(0, \beta)=\left(u_{2}-\beta\left(u_{2}-u_{1}\right)\right) v_{1}, \\
& y_{2}^{\prime}(0, \beta)=\left(u_{3}+\beta\left(u_{4}-u_{3}\right)\right) v_{1}, \\
& y_{1}^{\prime \prime}(0, \gamma)=\left(u_{2}-\gamma\left(u_{2}-u_{1}\right)\right) \kappa_{1}, \\
& y_{2}^{\prime \prime}(0, \gamma)=\left(u_{3}+\gamma\left(u_{4}-u_{3}\right)\right) \kappa_{1} \\
& y_{1}(l, \alpha)=\left(v_{1}+\alpha\left(v_{2}-v_{1}\right)\right) \vartheta_{2}, \\
& y_{2}(l, \alpha)=\left(v_{4}-\alpha\left(v_{4}-v_{3}\right)\right) \vartheta_{2}, \\
& y_{1}^{\prime}(l, \beta)=\left(v_{2}-\beta\left(v_{2}-v_{1}\right)\right) v_{2}, \\
& y_{2}^{\prime}(l, \beta)=\left(v_{3}+\beta\left(v_{4}-v_{3}\right)\right) v_{2}, \\
& y_{1}^{\prime \prime}(l, \gamma)=\left(v_{2}-\gamma\left(v_{2}-v_{1}\right)\right) \kappa_{2}, \\
& y_{2}^{\prime \prime}(l, \gamma)=\left(v_{3}+\gamma\left(v_{4}-v_{3}\right)\right) \kappa_{2} .
\end{aligned}
$$

\section{Solution}

The general solution of the first equation is as follows:

$y_{1}(x, \alpha)=k_{1} e^{\sqrt{p} x}+k_{2} e^{-\sqrt{p} x}$.

Applying the boundary conditions, we get $k_{1}+k_{2}=\left(u_{1}+\right.$ $\left.\alpha\left(u_{2}-u_{1}\right)\right) \vartheta_{1}$ and $k_{1} e^{\sqrt{p} l}+k_{2} e^{-\sqrt{p} l}=\left(v_{1}+\alpha\left(v_{2}-v_{1}\right)\right) \vartheta_{2}$, and solving the above, we have the following:

$$
\begin{aligned}
k_{1}= & \frac{1}{e^{\sqrt{p} l}-e^{-\sqrt{p} l}}\left\{\left[\left(v_{1}+\alpha\left(v_{2}-v_{1}\right)\right) \vartheta_{2}\right]\right. \\
& \left.-\left[\left(u_{1}+\alpha\left(u_{2}-u_{1}\right)\right) \vartheta_{1}\right] e^{-\sqrt{p} l}\right\}
\end{aligned}
$$

and

$$
\begin{aligned}
k_{2}= & -\frac{1}{e^{\sqrt{p} l}-e^{-\sqrt{p} l}}\left\{\left[\left(v_{1}+\alpha\left(v_{2}-v_{1}\right)\right) \vartheta_{2}\right]\right. \\
& \left.-\left[\left(u_{1}+\alpha\left(u_{2}-u_{1}\right)\right) \vartheta_{1}\right] e^{\sqrt{p} l}\right\} .
\end{aligned}
$$

Therefore, the general solution is as follows:

$$
\begin{aligned}
y_{1}(x, \alpha)= & \frac{1}{e^{\sqrt{p} l}-e^{-\sqrt{p} l}}\left\{\left(\left[\left(v_{1}+\alpha\left(v_{2}-v_{1}\right)\right) \vartheta_{2}\right]\right.\right. \\
& \left.-\left[\left(u_{1}+\alpha\left(u_{2}-u_{1}\right)\right) \vartheta_{1}\right] e^{-\sqrt{p} l}\right) e^{\sqrt{p} x} \\
& -\left(\left[\left(v_{1}+\alpha\left(v_{2}-v_{1}\right)\right) \vartheta_{2}\right]\right. \\
& \left.\left.-\left[\left(u_{1}+\alpha\left(u_{2}-u_{1}\right)\right) \vartheta_{1}\right] e^{\sqrt{p} l}\right) e^{-\sqrt{p} x}\right\} .
\end{aligned}
$$

Similarly

$$
\begin{aligned}
y_{2}(x, \alpha)= & \frac{1}{e^{\sqrt{p} l}-e^{-\sqrt{p} l}}\left\{\left(\left[\left(v_{4}-\alpha\left(v_{4}-v_{3}\right)\right) \vartheta_{2}\right]\right.\right. \\
& \left.-\left[\left(u_{4}-\alpha\left(u_{4}-u_{3}\right)\right) \vartheta_{1}\right] e^{-\sqrt{p} l}\right) e^{\sqrt{p} x} \\
& -\left(\left[\left(v_{4}-\alpha\left(v_{4}-v_{3}\right)\right) \vartheta_{2}\right]\right. \\
& \left.\left.-\left[\left(u_{4}-\alpha\left(u_{4}-u_{3}\right)\right) \vartheta_{1}\right] e^{\sqrt{p} l}\right) e^{-\sqrt{p} x}\right\}
\end{aligned}
$$

$$
\begin{aligned}
y_{1}^{\prime}(x, \beta)= & \frac{1}{e^{\sqrt{p} l}-e^{-\sqrt{p} l}}\left\{\left(\left[\left(v_{2}-\beta\left(v_{2}-v_{1}\right)\right) \nu_{2}\right]\right.\right. \\
& \left.-\left[\left(u_{2}-\beta\left(u_{2}-u_{1}\right)\right) v_{1}\right] e^{-\sqrt{p} l}\right) e^{\sqrt{p} x} \\
& -\left(\left[\left(v_{2}-\beta\left(v_{2}-v_{1}\right)\right) v_{2}\right]\right. \\
& \left.\left.-\left[\left(u_{2}-\beta\left(u_{2}-u_{1}\right)\right) v_{1}\right] e^{\sqrt{p} l}\right) e^{-\sqrt{p} x}\right\} \\
y_{2}^{\prime}(x, \beta)= & \frac{1}{e^{\sqrt{p} l}-e^{-\sqrt{p} l}}\left\{\left(\left[\left(v_{3}+\beta\left(v_{4}-v_{3}\right)\right) \nu_{2}\right]\right.\right. \\
& \left.-\left[\left(u_{3}+\beta\left(u_{4}-u_{3}\right)\right) v_{1}\right] e^{-\sqrt{p} l}\right) e^{\sqrt{p} x} \\
& -\left(\left[\left(v_{3}+\beta\left(v_{4}-v_{3}\right)\right) v_{2}\right]\right. \\
& \left.\left.-\left[\left(u_{3}+\beta\left(u_{4}-u_{3}\right)\right) v_{1}\right] e^{\sqrt{p} l}\right) e^{-\sqrt{p} x}\right\} \\
y_{1}^{\prime \prime}(x, \gamma)= & \frac{1}{e^{\sqrt{p} l}-e^{-\sqrt{p} l}}\left\{\left(\left[\left(v_{2}-\gamma\left(v_{2}-v_{1}\right)\right) \kappa_{2}\right]\right.\right. \\
& \left.-\left[\left(u_{2}-\gamma\left(u_{2}-u_{1}\right)\right) \kappa_{1}\right] e^{-\sqrt{p} l}\right) e^{\sqrt{p} x} \\
& -\left(\left[\left(v_{2}-\gamma\left(v_{2}-v_{1}\right)\right) \kappa_{2}\right]\right. \\
& \left.\left.-\left[\left(u_{2}-\gamma\left(u_{2}-u_{1}\right)\right) \kappa_{1}\right] e^{\sqrt{p} l}\right) e^{-\sqrt{p} x}\right\} \\
y_{2}^{\prime \prime}(x, \gamma)= & \frac{1}{e^{\sqrt{p} l}-e^{-\sqrt{p} l}\left\{\left(\left[\left(v_{3}+\gamma\left(v_{4}-v_{3}\right)\right) \kappa_{2}\right]\right.\right.} \\
& \left.-\left[\left(u_{3}+\gamma\left(u_{4}-u_{3}\right)\right) \kappa_{1}\right] e^{-\sqrt{p} l}\right) e^{\sqrt{p} x} \\
& -\left(\left[\left(v_{3}+\gamma\left(v_{4}-v_{3}\right)\right) \kappa_{2}\right]\right. \\
& \left.\left.-\left[\left(u_{3}+\gamma\left(u_{4}-u_{3}\right)\right) \kappa_{1}\right] e^{\sqrt{p} l}\right) e^{-\sqrt{p} x}\right\} .
\end{aligned}
$$

Case 2: $y(x)$ is $\mathscr{D}^{2}$-differentiable and $\frac{\mathrm{d} y(x)}{\mathrm{d} x}$ is $\mathscr{D}^{1}$-differentiable or $y(x)$ is $\mathscr{D}^{1}$-differentiable and $\frac{\mathrm{d} y(x)}{\mathrm{d} x}$ is $\mathscr{D}^{1}$-differentiable, and then, we have the following:

$$
\begin{array}{ll}
\frac{\mathrm{d}^{2} y_{1}(x, \alpha)}{\mathrm{d} x^{2}}=p y_{2}(x, \alpha) ; & \frac{\mathrm{d}^{2} y_{2}(x, \alpha)}{\mathrm{d} x^{2}}=p y_{1}(x, \alpha) \\
\frac{\mathrm{d}^{2} y_{1}^{\prime}(x, \beta)}{\mathrm{d} x^{2}}=p y_{2}^{\prime}(x, \beta) ; & \frac{\mathrm{d}^{2} y_{2}^{\prime}(x, \beta)}{\mathrm{d} x^{2}}=p y_{1}^{\prime}(x, \alpha) \\
\frac{\mathrm{d}^{2} y_{1}^{\prime \prime}(x, \gamma)}{\mathrm{d} x^{2}}=p y_{2}^{\prime \prime}(x, \gamma) ; & \frac{\mathrm{d}^{2} y_{2}^{\prime \prime}(x, \gamma)}{\mathrm{d} x^{2}}=p y_{1}^{\prime \prime}(x, \gamma)
\end{array}
$$

with the boundary conditions (1).

The general solution is as follows:

$$
\begin{aligned}
& y_{1}(x, \alpha)=k_{1} e^{\sqrt{p} x}+k_{2} e^{-\sqrt{p} x}+k_{3} \cos \sqrt{p} x+k_{4} \sin \sqrt{p} x \\
& y_{2}(x, \alpha)=k_{1} e^{\sqrt{p} x}+k_{2} e^{-\sqrt{p} x}-k_{3} \cos \sqrt{p} x-k_{4} \sin \sqrt{p} x \\
& y_{1}^{\prime}(x, \beta)=k_{5} e^{\sqrt{p} x}+k_{6} e^{-\sqrt{p} x}+k_{7} \cos \sqrt{p} x+k_{8} \sin \sqrt{p} x \\
& y_{2}^{\prime}(x, \beta)=k_{5} e^{\sqrt{p} x}+k_{6} e^{-\sqrt{p} x}-k_{7} \cos \sqrt{p} x-k_{8} \sin \sqrt{p} x \\
& y_{1}^{\prime \prime}(x, \gamma)=k_{9} e^{\sqrt{p} x}+k_{10} e^{-\sqrt{p} x}+k_{11} \cos \sqrt{p} x+k_{12} \sin \sqrt{p} x \\
& y_{2}^{\prime \prime}(x, \gamma)=k_{9} e^{\sqrt{p} x}+k_{10} e^{-\sqrt{p} x}-k_{11} \cos \sqrt{p} x-k_{12} \sin \sqrt{p} x,
\end{aligned}
$$

where

$$
\begin{aligned}
k_{1}= & \frac{1}{e^{\sqrt{p} l}-e^{-\sqrt{p} l}}\left\{\left[\left(\frac{\left(v_{1}+v_{4}\right)+\alpha\left(\left(v_{2}-v_{1}\right)-\left(v_{4}-v_{3}\right)\right)}{2}\right) \vartheta_{2}\right]\right. \\
& \left.-\left[\left(\frac{\left(u_{1}+u_{4}\right)+\alpha\left(\left(u_{2}-u_{1}\right)-\left(u_{4}-u_{3}\right)\right)}{2}\right) \vartheta_{1}\right] e^{-\sqrt{p} l}\right\} \\
k_{2}= & \frac{-1}{e^{\sqrt{p} l}-e^{-\sqrt{p} l}}\left\{\left[\left(\frac{\left(v_{1}+v_{4}\right)+\alpha\left(\left(v_{2}-v_{1}\right)-\left(v_{4}-v_{3}\right)\right)}{2}\right) \vartheta_{2}\right]\right.
\end{aligned}
$$




$$
\begin{aligned}
& \left.-\left[\left(\frac{\left(u_{1}+u_{4}\right)+\alpha\left(\left(u_{2}-u_{1}\right)-\left(u_{4}-u_{3}\right)\right)}{2}\right) \vartheta_{1}\right] e^{\sqrt{p} l}\right\} \\
& k_{3}=\left[\frac{\left(u_{1}-u_{4}\right)}{2}+\left(\frac{\alpha\left(\left(v_{2}-v_{1}\right)+\left(v_{4}-v_{3}\right)\right)}{2}\right) \vartheta_{2}\right] \\
& k_{4}=\frac{1}{\sin \sqrt{p} l}\left\{\left[\left(\frac{\left(v_{1}+v_{4}\right)+\alpha\left(\left(v_{2}-v_{1}\right)-\left(v_{4}-v_{3}\right)\right)}{2}\right) \vartheta_{2}\right]\right. \\
& \left.-\left[\left(\frac{\left(u_{1}+u_{4}\right)+\alpha\left(\left(u_{2}-u_{1}\right)-\left(u_{4}-u_{3}\right)\right)}{2}\right) \vartheta_{1}\right] \cos \sqrt{p} l\right\} \\
& k_{5}=\frac{1}{e^{\sqrt{p} l}-e^{-\sqrt{p} l}}\left\{\left[\left(\frac{\left(v_{2}+v_{3}\right)-\beta\left(\left(v_{2}-v_{1}\right)-\left(v_{4}-v_{3}\right)\right)}{2}\right) v_{2}\right]\right. \\
& \left.-\left[\left(\frac{\left(u_{2}+u_{3}\right)-\beta\left(\left(u_{2}-u_{1}\right)-\left(u_{4}-u_{3}\right)\right)}{2}\right) v_{1}\right] e^{-\sqrt{p} l}\right\} \\
& k_{6}=\frac{-1}{e^{\sqrt{p} l}-e^{-\sqrt{p} l}}\left\{\left[\left(\frac{\left(v_{2}+v_{3}\right)-\beta\left(\left(v_{2}-v_{1}\right)-\left(v_{4}-v_{3}\right)\right)}{2}\right) v_{2}\right]\right. \\
& \left.-\left[\left(\frac{\left(u_{2}+u_{3}\right)-\beta\left(\left(u_{2}-u_{1}\right)-\left(u_{4}-u_{3}\right)\right)}{2}\right) v_{1}\right] e^{\sqrt{p} l}\right\} \\
& k_{7}=\left[\frac{\left(u_{2}-u_{3}\right)}{2}-\left(\frac{\beta\left(\left(v_{2}-v_{1}\right)+\left(v_{4}-v_{3}\right)\right)}{2}\right) v_{2}\right] \\
& k_{8}=\frac{1}{\sin \sqrt{p} l}\left\{\left[\left(\frac{\left(v_{2}-v_{3}\right)-\beta\left(\left(v_{2}-v_{1}\right)-\left(v_{4}-v_{3}\right)\right)}{2}\right) v_{2}\right]\right. \\
& \left.-\left[\left(\frac{\left(u_{2}-u_{3}\right)-\beta\left(\left(u_{2}-u_{1}\right)+\left(u_{4}-u_{3}\right)\right)}{2}\right) v_{1}\right] \cos \sqrt{p} l\right\} \\
& k_{9}=\frac{1}{e^{\sqrt{p} l}-e^{-\sqrt{p} l}}\left\{\left[\left(\frac{\left(v_{2}+v_{3}\right)-\gamma\left(\left(v_{2}-v_{1}\right)-\left(v_{4}-v_{3}\right)\right)}{2}\right) \kappa_{2}\right]\right. \\
& \left.-\left[\left(\frac{\left(u_{2}+u_{3}\right)-\gamma\left(\left(u_{2}-u_{1}\right)-\left(u_{4}-u_{3}\right)\right)}{2}\right) \kappa_{1}\right] e^{-\sqrt{p} l}\right\} \\
& k_{10}=\frac{-1}{e^{\sqrt{p} l}-e^{-\sqrt{p} l}}\left\{\left[\left(\frac{\left(v_{2}+v_{3}\right)-\gamma\left(\left(v_{2}-v_{1}\right)-\left(v_{4}-v_{3}\right)\right)}{2}\right) \kappa_{2}\right]\right. \\
& \left.-\left[\left(\frac{\left(u_{2}+u_{3}\right)-\gamma\left(\left(u_{2}-u_{1}\right)-\left(u_{4}-u_{3}\right)\right)}{2}\right) \kappa_{1}\right] e^{\sqrt{p} l}\right\} \\
& k_{11}=\left[\frac{\left(u_{2}-u_{3}\right)}{2}-\left(\frac{\gamma\left(\left(v_{2}-v_{1}\right)+\left(v_{4}-v_{3}\right)\right)}{2}\right) \kappa_{2}\right] \\
& k_{12}=\frac{1}{\sin \sqrt{p} l}\left\{\left[\left(\frac{\left(v_{2}-v_{3}\right)-\gamma\left(\left(v_{2}-v_{1}\right)-\left(v_{4}-v_{3}\right)\right)}{2}\right) \kappa_{2}\right]\right. \\
& \left.-\left[\left(\frac{\left(u_{2}-u_{3}\right)-\gamma\left(\left(u_{2}-u_{1}\right)+\left(u_{4}-u_{3}\right)\right)}{2}\right) \kappa_{1}\right] \cos \sqrt{p} l\right\} \text {. }
\end{aligned}
$$

2. When $p$ is negative constant, i.e., $p<0$, let $p=-q$ and $q>0$, then two cases are possible.

Case 1: $y(x)$ and $\frac{\mathrm{d} y(x)}{\mathrm{d} x}$ are $\mathscr{D}^{1}$-differentiable or $\mathscr{D}^{2}$-differen tiable, and then, we have the following:

$$
\begin{array}{ll}
\frac{\mathrm{d}^{2} y_{1}(x, \alpha)}{\mathrm{d} x^{2}}=-q y_{1}(x, \alpha) ; & \frac{\mathrm{d}^{2} y_{2}(x, \alpha)}{\mathrm{d} x^{2}}=-q y_{2}(x, \alpha) \\
\frac{\mathrm{d}^{2} y_{1}^{\prime}(x, \beta)}{\mathrm{d} x^{2}}=-q y_{1}^{\prime}(x, \beta) ; & \frac{\mathrm{d}^{2} y_{2}^{\prime}(x, \beta)}{\mathrm{d} x^{2}}=-q y_{2}^{\prime}(x, \beta) \\
\frac{\mathrm{d}^{2} y_{1}^{\prime \prime}(x, \gamma)}{\mathrm{d} x^{2}}=-q y_{1}^{\prime \prime}(x, \gamma) ; & \frac{\mathrm{d}^{2} y_{2}^{\prime \prime}(x, \gamma)}{\mathrm{d} x^{2}}=-q y_{2}^{\prime \prime}(x, \gamma)
\end{array}
$$

with the boundary conditions (1).
The general solution of the above equations are as follows:

$y_{1}(x, \alpha)=k_{1} e^{\sqrt{q} x}+k_{2} e^{-\sqrt{q} x}+k_{3} \cos \sqrt{q} x+k_{4} \sin \sqrt{q} x$ $y_{2}(x, \alpha)=-k_{1} e^{\sqrt{q} x}-k_{2} e^{-\sqrt{q} x}+k_{3} \cos \sqrt{q} x+k_{4} \sin \sqrt{q} x$ $y_{1}^{\prime}(x, \beta)=k_{5} e^{\sqrt{q} x}+k_{6} e^{-\sqrt{q} x}+k_{7} \cos \sqrt{q} x+k_{8} \sin \sqrt{q} x$ $y_{2}^{\prime}(x, \beta)=-k_{5} e^{\sqrt{q} x}-k_{6} e^{-\sqrt{q} x}+k_{7} \cos \sqrt{q} x+k_{8} \sin \sqrt{q} x$ $y_{1}^{\prime \prime}(x, \gamma)=k_{9} e^{\sqrt{q} x}+k_{10} e^{-\sqrt{q} x}+k_{11} \cos \sqrt{q} x+k_{12} \sin \sqrt{q} x$ $y_{2}^{\prime \prime}(x, \gamma)=-k_{9} e^{\sqrt{q} x}-k_{10} e^{-\sqrt{q} x}+k_{11} \cos \sqrt{q} x+k_{12} \sin \sqrt{q} x$,

where

$$
\begin{aligned}
& k_{1}=\frac{1}{e^{\sqrt{q} l}-e^{-\sqrt{q} l}}\left\{\left[\left(\frac{\left(v_{1}-v_{4}\right)+\alpha\left(\left(v_{2}-v_{1}\right)+\left(v_{4}-v_{3}\right)\right)}{2}\right) \vartheta_{2}\right]\right. \\
& \left.-\left[\left(\frac{\left(u_{1}-u_{4}\right)+\alpha\left(\left(u_{2}-u_{1}\right)+\left(u_{4}-u_{3}\right)\right)}{2}\right) \vartheta_{1}\right] e^{-\sqrt{q} l}\right\} \\
& k_{2}=\frac{-1}{e^{\sqrt{q} l}-e^{-\sqrt{q} l}}\left\{\left[\left(\frac{\left(v_{1}-v_{4}\right)+\alpha\left(\left(v_{2}-v_{1}\right)+\left(v_{4}-v_{3}\right)\right)}{2}\right) \vartheta_{2}\right]\right. \\
& \left.-\left[\left(\frac{\left(u_{1}-u_{4}\right)+\alpha\left(\left(u_{2}-u_{1}\right)+\left(u_{4}-u_{3}\right)\right)}{2}\right) \vartheta_{1}\right] e^{\sqrt{q} l}\right\} \\
& k_{3}=\left[\frac{\left(u_{1}+u_{4}\right)}{2}+\left(\frac{\alpha\left(\left(v_{2}-v_{1}\right)-\left(v_{4}-v_{3}\right)\right)}{2}\right) \vartheta_{2}\right] \\
& k_{4}=\frac{1}{\sin \sqrt{q} l}\left\{\left[\left(\frac{\left(v_{1}+v_{4}\right)+\alpha\left(\left(v_{2}-v_{1}\right)-\left(v_{4}-v_{3}\right)\right)}{2}\right) \vartheta_{2}\right]\right. \\
& \left.-\left[\left(\frac{\left(u_{1}+u_{4}\right)+\alpha\left(\left(u_{2}-u_{1}\right)-\left(u_{4}-u_{3}\right)\right)}{2}\right) \vartheta_{1}\right] \cos \sqrt{q} l\right\} \\
& k_{5}=\frac{1}{e^{\sqrt{q} l}-e^{-\sqrt{q} l}}\left\{\left[\left(\frac{\left(v_{2}-v_{3}\right)-\beta\left(\left(v_{2}-v_{1}\right)+\left(v_{4}-v_{3}\right)\right)}{2}\right) v_{2}\right]\right. \\
& \left.-\left[\left(\frac{\left(u_{2}-u_{3}\right)-\beta\left(\left(u_{2}-u_{1}\right)+\left(u_{4}-u_{3}\right)\right)}{2}\right) v_{1}\right] e^{-\sqrt{q} l}\right\} \\
& k_{6}=\frac{-1}{e^{\sqrt{q} l}-e^{-\sqrt{q} l}}\left\{\left[\left(\frac{\left(v_{2}-v_{3}\right)-\beta\left(\left(v_{2}-v_{1}\right)+\left(v_{4}-v_{3}\right)\right)}{2}\right) v_{2}\right]\right. \\
& \left.-\left[\left(\frac{\left(u_{2}-u_{3}\right)-\beta\left(\left(u_{2}-u_{1}\right)+\left(u_{4}-u_{3}\right)\right)}{2}\right) v_{1}\right] e^{\sqrt{q} l}\right\} \\
& k_{7}=\left[\frac{\left(u_{2}+u_{3}\right)}{2}-\left(\frac{\beta\left(\left(v_{2}-v_{1}\right)-\left(v_{4}-v_{3}\right)\right)}{2}\right) v_{2}\right] \\
& k_{8}=\frac{1}{\sin \sqrt{q} l}\left\{\left[\left(\frac{\left(v_{2}+v_{3}\right)-\beta\left(\left(v_{2}-v_{1}\right)-\left(v_{4}-v_{3}\right)\right)}{2}\right) v_{2}\right]\right. \\
& \left.-\left[\left(\frac{\left(u_{2}+u_{3}\right)-\beta\left(\left(u_{2}-u_{1}\right)-\left(u_{4}-u_{3}\right)\right)}{2}\right) v_{1}\right] \cos \sqrt{q} l\right\} \\
& k_{9}=\frac{1}{e^{\sqrt{q} l}-e^{-\sqrt{q} l}}\left\{\left[\left(\frac{\left(v_{2}-v_{3}\right)-\gamma\left(\left(v_{2}-v_{1}\right)+\left(v_{4}-v_{3}\right)\right)}{2}\right) \kappa_{2}\right]\right. \\
& \left.-\left[\left(\frac{\left(u_{2}-u_{3}\right)-\gamma\left(\left(u_{2}-u_{1}\right)+\left(u_{4}-u_{3}\right)\right)}{2}\right) \kappa_{1}\right] e^{-\sqrt{q} l}\right\} \\
& k_{10}=\frac{-1}{e^{\sqrt{q} l}-e^{-\sqrt{q} l}}\left\{\left[\left(\frac{\left(v_{2}-v_{3}\right)-\gamma\left(\left(v_{2}-v_{1}\right)+\left(v_{4}-v_{3}\right)\right)}{2}\right) \kappa_{2}\right]\right. \\
& \left.-\left[\left(\frac{\left(u_{2}-u_{3}\right)-\gamma\left(\left(u_{2}-u_{1}\right)+\left(u_{4}-u_{3}\right)\right)}{2}\right) \kappa_{1}\right] e^{\sqrt{q} l}\right\} \\
& k_{11}=\left[\frac{\left(u_{2}+u_{3}\right)}{2}-\left(\frac{\gamma\left(\left(v_{2}-v_{1}\right)-\left(v_{4}-v_{3}\right)\right)}{2}\right) \kappa_{2}\right] \\
& k_{12}=\frac{1}{\sin \sqrt{q} l}\left\{\left[\left(\frac{\left(v_{2}+v_{3}\right)-\gamma\left(\left(v_{2}-v_{1}\right)-\left(v_{4}-v_{3}\right)\right)}{2}\right) \kappa_{2}\right]\right. \\
& \left.-\left[\left(\frac{\left(u_{2}+u_{3}\right)-\gamma\left(\left(u_{2}-u_{1}\right)-\left(u_{4}-u_{3}\right)\right)}{2}\right) \kappa_{1}\right] \cos \sqrt{q} l\right\} \text {. }
\end{aligned}
$$


Case 2: $y(x)$ is $\mathscr{D}^{1}$-differentiable and $\frac{\mathrm{d} y(x)}{\mathrm{d} x}$ is $\mathscr{D}^{2}$-differentiable or $y(x)$ is $\mathscr{D}^{2}$-differentiable and $\frac{\mathrm{d} y(x)}{\mathrm{d} x}$ is $\mathscr{D}^{1}$-differentiable

$$
\begin{array}{ll}
\frac{\mathrm{d}^{2} y_{1}(x, \alpha)}{\mathrm{d} x^{2}}=-q y_{2}(x, \alpha) ; & \frac{\mathrm{d}^{2} y_{2}(x, \alpha)}{\mathrm{d} x^{2}}=-q y_{1}(x, \alpha) \\
\frac{\mathrm{d}^{2} y_{1}^{\prime}(x, \beta)}{\mathrm{d} x^{2}}=-q y_{2}^{\prime}(x, \beta) ; & \frac{\mathrm{d}^{2} y_{2}^{\prime}(x, \beta)}{\mathrm{d} x^{2}}=-q y_{1}^{\prime}(x, \beta) \\
\frac{\mathrm{d}^{2} y_{1}^{\prime \prime}(x, \gamma)}{\mathrm{d} x^{2}}=-q y_{2}^{\prime \prime}(x, \gamma) ; & \frac{\mathrm{d}^{2} y_{2}^{\prime \prime}(x, \gamma)}{\mathrm{d} x^{2}}=-q y_{1}^{\prime \prime}(x, \gamma)
\end{array}
$$

with the boundary conditions (1), and then, the solution is as follows:

$$
\begin{aligned}
y_{1}(x, \alpha)= & {\left[\left(u_{1}+\alpha\left(u_{2}-u_{1}\right)\right) \vartheta_{1}\right] \cos \sqrt{q} l } \\
& +\frac{1}{\sin \sqrt{q} l}\left\{\left[\left(v_{1}+\alpha\left(v_{2}-v_{1}\right)\right) \vartheta_{2}\right]\right. \\
& \left.-\left[\left(u_{1}+\alpha\left(u_{2}-u_{1}\right)\right) \vartheta_{1}\right] \cos \sqrt{q} l\right\} \sin \sqrt{q} x \\
y_{2}(x, \alpha)= & {\left[\left(u_{4}-\alpha\left(u_{4}-u_{3}\right)\right) \vartheta_{1}\right] \cos \sqrt{q} l } \\
& +\frac{1}{\sin \sqrt{q} l}\left\{\left[\left(v_{4}-\alpha\left(v_{4}-v_{3}\right)\right) \vartheta_{2}\right]\right. \\
& \left.-\left[\left(u_{4}-\alpha\left(u_{4}-u_{3}\right)\right) \vartheta_{1}\right] \cos \sqrt{q} l\right\} \sin \sqrt{q} x \\
y_{1}^{\prime}(x, \beta)= & {\left[\left(u_{3}+\beta\left(u_{4}-u_{3}\right)\right) v_{1}\right] \cos \sqrt{q} l } \\
& +\frac{1}{\sin \sqrt{q} l}\left\{\left[\left(v_{2}-\beta\left(v_{2}-v_{1}\right)\right) v_{2}\right]\right. \\
& \left.-\left[\left(u_{3}+\beta\left(u_{4}-u_{3}\right)\right) v_{1}\right] \cos \sqrt{q} l\right\} \sin \sqrt{q} x \\
y_{2}^{\prime}(x, \beta)= & {\left[\left(u_{2}-\beta\left(u_{2}-u_{1}\right)\right) v_{1}\right] \cos \sqrt{q} l } \\
& +\frac{1}{\sin \sqrt{q} l}\left\{\left[\left(v_{3}+\beta\left(v_{4}-v_{3}\right)\right) v_{2}\right]\right. \\
& \left.-\left[\left(u_{2}-\beta\left(u_{2}-u_{1}\right)\right) v_{1}\right] \cos \sqrt{q} l\right\} \sin \sqrt{q} x \\
& \left.-\left[\left(u_{3}+\gamma\left(u_{4}-u_{3}\right)\right) \kappa_{1}\right] \cos \sqrt{q} l\right\} \sin \sqrt{q} x \\
& +\frac{1}{\sin \sqrt{q} l}\left\{\left[\left(v_{2}-\gamma\left(v_{2}-v_{1}\right)\right) \kappa_{2}\right]\right. \\
y_{1}^{\prime \prime}(x, \gamma)= & {\left[\left(u_{3}+\gamma\left(u_{4}-u_{3}\right)\right) \kappa_{1}\right] \cos \sqrt{q} l } \\
&
\end{aligned}
$$

$$
\begin{aligned}
y_{2}^{\prime \prime}(x, \gamma)= & {\left[\left(u_{2}-\gamma\left(u_{2}-u_{1}\right)\right) \kappa_{1}\right] \cos \sqrt{q} l } \\
& +\frac{1}{\sin \sqrt{q} l}\left\{\left[\left(v_{3}+\gamma\left(v_{4}-v_{3}\right)\right) \kappa_{2}\right]\right. \\
& \left.-\left[\left(u_{2}-\gamma\left(u_{2}-u_{1}\right)\right) \kappa_{1}\right] \cos \sqrt{q} l\right\} \sin \sqrt{q} x .
\end{aligned}
$$

\section{Analytic example}

Let us consider the differential equation $\frac{\mathrm{d}^{2} y(x)}{\mathrm{d} x^{2}}=y(x)$ with the boundary conditions $y(0)=((3,4,5,6) ; 0.7,0.6,0.4)$ and $y(2)=((7,8,9,10) ; 0.4,0.5,0.6)$, and let $\mathrm{y}(\mathrm{x})$ and $\frac{\mathrm{d} y(x)}{\mathrm{d} x}$ is $\mathscr{D}^{1}$-differentiable.

\section{Solution}

$$
\begin{aligned}
y_{1}(x, \alpha)= & \frac{1}{e^{2}-e^{-2}}\left\{\left[(7+0.4 \alpha)-(3+0.7 \alpha) e^{-2}\right] e\right. \\
& \left.-\left[(7+0.4 \alpha)-(3+0.7 \alpha) e^{2}\right] e^{-1}\right\} \\
y_{2}(x, \alpha)= & \frac{1}{e^{2}-e^{-2}}\left\{\left[(10-0.4 \alpha)-(6-0.7 \alpha) e^{-2}\right] e\right. \\
& \left.-\left[(10-0.4 \alpha)-(6-0.7 \alpha) e^{2}\right] e^{-1}\right\} \\
y_{1}^{\prime}(x, \beta)= & \frac{1}{e^{2}-e^{-2}}\left\{\left[(8-0.5 \beta)-(4-0.6 \beta) e^{-2}\right] e\right. \\
& \left.-\left[(8-0.5 \beta)-(4-0.6 \beta) e^{2}\right] e^{-1}\right\} \\
y_{2}^{\prime}(x, \beta)= & \frac{1}{e^{2}-e^{-2}}\left\{\left[(9+0.5 \beta)-(5+0.6 \beta) e^{-2}\right] e\right. \\
& \left.-\left[(9+0.5 \beta)-(5+0.6 \beta) e^{2}\right] e^{-1}\right\} \\
& \left.-\left[(8-0.6 \gamma)-(4-0.4 \gamma) e^{2}\right] e^{-1}\right\} \\
y_{1}^{\prime \prime}(x, \gamma)= & \frac{1}{e^{2}-e^{-2}}\left\{\left[(8-0.6 \gamma)-(4-0.4 \gamma) e^{-2}\right] e\right. \\
& \left.-\left[(9+0.6 \gamma)-(5+0.4 \gamma) e^{2}\right] e^{-1}\right\} . \\
y_{2}^{\prime \prime}(x, \gamma)= & \frac{1}{e^{2}-e^{-2}}\left\{\left[(9+0.6 \gamma)-(5+0.4 \gamma) e^{-2}\right] e\right. \\
& -[(9)+1
\end{aligned}
$$

Table 1 Solution for $x=1$

\begin{tabular}{lllllllll}
\hline$\alpha$ & $y_{1}(x, \alpha)$ & $y_{2}(x, \alpha)$ & $\beta$ & $y_{1}^{\prime}(x, \beta)$ & $y_{2}^{\prime}(x, \beta)$ & $\gamma$ & $y_{1}^{\prime \prime}(x, \gamma)$ & $y_{2}^{\prime \prime}(x, \gamma)$ \\
\hline 0 & 3.2403 & 6.4805 & 0 & 3.8883 & 4.5364 & 0 & 3.8883 & 4.5364 \\
0.1 & 3.2759 & 6.4449 & 0.1 & 3.8527 & 4.572 & 0.1 & 3.8559 & 4.5688 \\
0.2 & 3.3116 & 6.4093 & 0.2 & 3.817 & 4.6077 & 0.2 & 3.8235 & 4.6012 \\
0.3 & 3.3472 & 6.3736 & 0.3 & 3.7814 & 4.6433 & 0.3 & 3.7911 & 4.6336 \\
0.4 & 3.3828 & 6.338 & 0.4 & 3.7458 & 4.679 & 0.4 & 3.7587 & 4.666 \\
0.5 & 3.4185 & 6.3023 & 0.5 & 3.7101 & 4.7146 & 0.5 & 3.7263 & 4.6984 \\
0.6 & 3.4541 & 6.2667 & 0.6 & 3.6745 & 4.7502 & 0.6 & 3.6939 & 4.7308 \\
0.7 & 3.4898 & 6.231 & 0.7 & 3.6388 & 4.7859 & 0.7 & 3.6615 & 4.7632 \\
0.8 & 3.5254 & 6.1954 & 0.8 & 3.6032 & 4.8215 & 0.8 & 3.6291 & 4.7956 \\
0.9 & 3.5611 & 6.1598 & 0.9 & 3.5675 & 4.8572 & 0.9 & 3.5967 & 4.828 \\
1 & 3.5967 & 6.1241 & 1 & 3.5319 & 4.8928 & 1 & 3.5643 & 4.8604 \\
\hline
\end{tabular}


When we take $x=1$ and for different values of $\alpha, \beta, \gamma$, the solution is given in Table 1 .

The graphical interpretation of the above table is shown in the following.
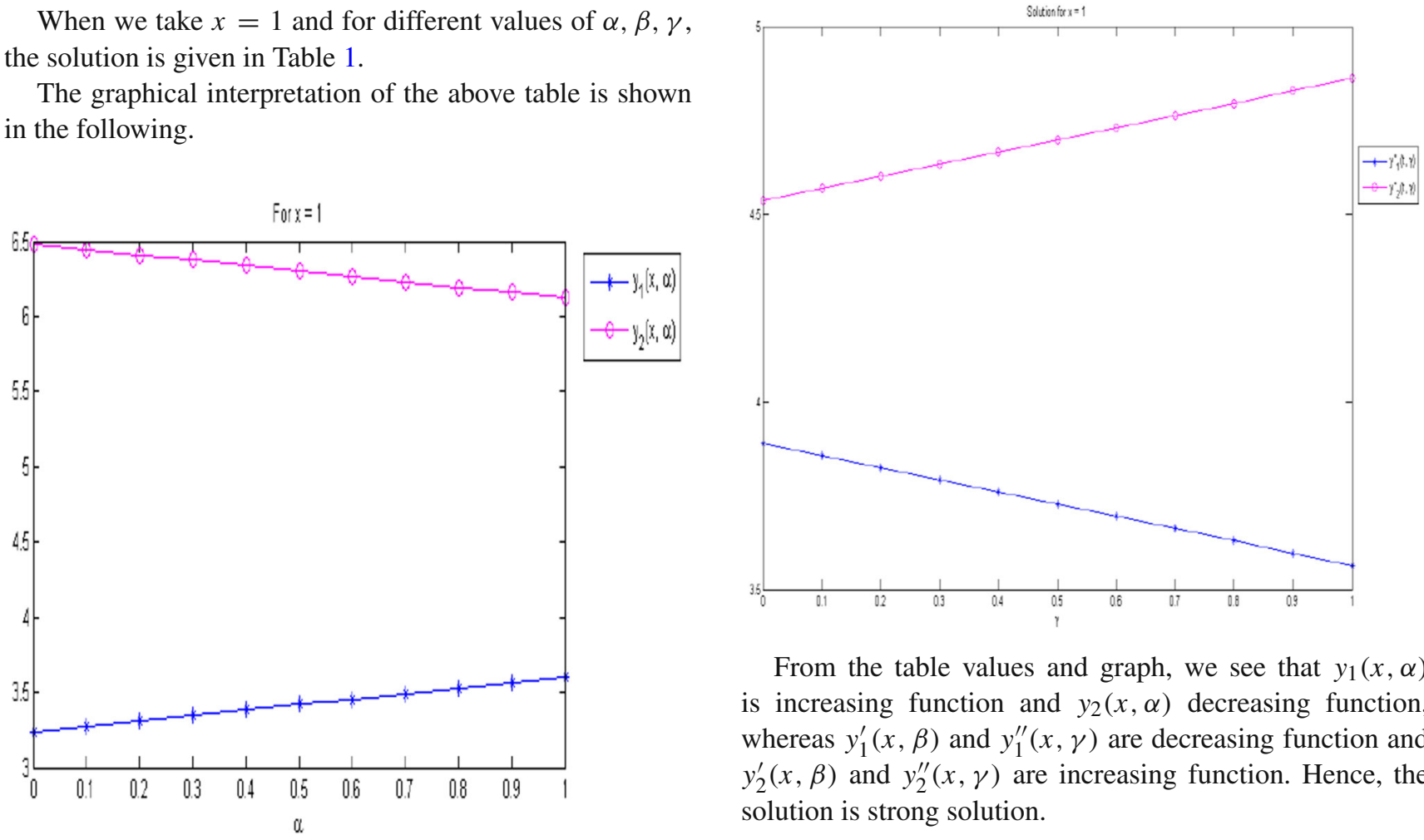

From the table values and graph, we see that $y_{1}(x, \alpha)$ is increasing function and $y_{2}(x, \alpha)$ decreasing function, whereas $y_{1}^{\prime}(x, \beta)$ and $y_{1}^{\prime \prime}(x, \gamma)$ are decreasing function and $y_{2}^{\prime}(x, \beta)$ and $y_{2}^{\prime \prime}(x, \gamma)$ are increasing function. Hence, the solution is strong solution.

\section{Conclusion}

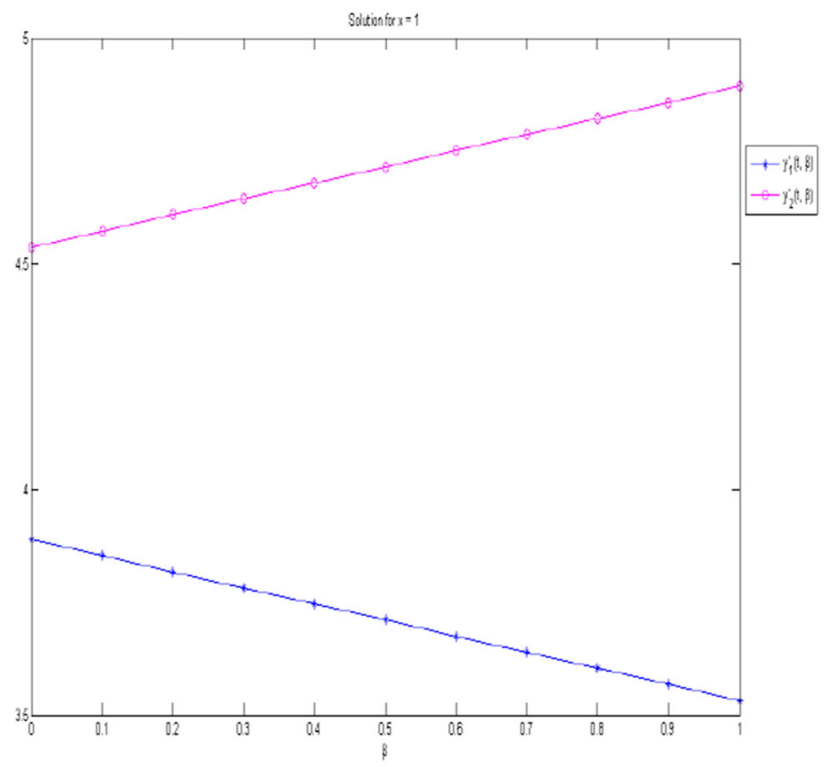

In this paper, we have derived the solution of second-order differential equation in neutrosophic environment. An example is given to demonstrate the strong solution of the same. For future research work, we use this approach to solve higher order differential equations and we can explore this method to solve linear and non-linear differential equations, simultaneous differential equations, and so on. Also the numerical techniques can be applied to solve the neutrosophic differential equations. Neutrosophic integration can be developed to solve the problems involving neutrosophic numbers in realworld applications. Internet of things is one of the developing areas in recent times. In [21], neutrosophic AHP (Analytical hierarchy process) of the IoT in enterprises has been effectively presented in decision-making criteria. Neutrosophic logic in IoT may be employed to handle inconsistent data.

Open Access This article is distributed under the terms of the Creative Commons Attribution 4.0 International License (http://creativecomm ons.org/licenses/by/4.0/), which permits unrestricted use, distribution, and reproduction in any medium, provided you give appropriate credit to the original author(s) and the source, provide a link to the Creative Commons license, and indicate if changes were made. 


\section{References}

1. Zadeh LA (1965) Fuzzy sets. Inf Control 8:338-353

2. Atanassov K (1986) Intuitionistic fuzzy sets. Fuzzy Sets Syst 20:87-96

3. Atanassov KT (1999) Intuitionistic fuzzy sets. Pysica-Verlag A Springer-Verlag Company, New York

4. Smarandache F (2002) Neutrosophy and neutrosophic logic, first international conference on neutrosophy, neutrosophic logic, set, probability, and statistics. University of New Mexico, Gallup, NM 87301, USA

5. Smarandache F (1998) A unifying field in logics. neutrosophy: neutrosophic probability, set and logic. American Research Press, Rehoboth

6. Smarandache F (2005) Neutrosophic set, a generalisation of the intuitionistic fuzzy sets. Int J Pure Appl Math 24:287-297

7. Wang H, Smarandache FY, Zhang Q, Sunderraman R (2010) Single valued neutrosophic sets. Multisp Multistruct 4:410-413

8. Introduction to neutrosophic measure, neutrosophic integral, and neutrosophic probability, by Florentin Smarandache, Sitech Educational, Craiova, Columbus, p 140 (2013)

9. Ye J (2015) Trapezoidal neutrosophic set and its application to multiple attribute decision-making. Neural Comput Appl 26:11571166

10. Deli I, Subas Y (2017) A ranking method of single valued neutrosophic numbers and its applications to multi-attribute decision making problems. Int J Mach Learn Cyber 8:1309-1322

11. Aal SIA, Abd Ellatif MMA, Hassan MM (2018) Proposed model for evaluating information systems quality based on single valued triangular neutrosophic numbers. IJ Math Sci Comput 1:1-14

12. Chakraborty A, Mondal SP, Ahmadian A, Senu N, Alam S, Salahshour S (2018) Different forms of triangular neutrosophic numbers, de-neutrosophication techniques, and their applications. Symmetry 10:327

13. Abdel-Basset M, Saleh M, Gamal A, Smarandache F (2019) An approach of TOPSIS technique for developing supplier selection with group decision making under type-2 neutrosophic number. Appl Soft Comput J 77:438-452

14. Abdel-Baset M, Chang V, Gamal A, Smarandache F (2019) An integrated neutrosophic ANP and VIKOR method for achieving sustainable supplier selection: a case study in importing field. Comput Ind 106:94-110

15. Abdel-Basset M, Manogaran G, Gamal A, Smarandache F (2019) A group decision making framework based on neutrosophic TOPSIS approach for smart medical device selection. J Med Syst 43:38

16. Abdel-Baset M, Atef A, Smarandache F (2019) A hybrid neutrosophic multiple criteria group decision making approach for project selection. Cognit Syst Res 57:216-227

17. Nabeeh NA, Smarandache F, Abdel-Basset M, El-Ghareeb HA, Aboelfetouh A (2019) An integrated neutrosophic-TOPSIS approach and its application to personnel selection: a new trend in brain processing and analysis. IEEE Access 7:29734-29744

18. Abdel-Baset M, Chang V, Gamal A (2019) Evaluation of the green supply chain management practices: a novel neutrosophic approach. Comput Ind 108:210-220

19. Abdel-Baset M, Manogaran G, Gamal A, Smarandache F (2018) A hybrid approach of neutrosophic sets and DEMATEL method for developing supplier selection criteria. Des Autom Embed Syst $22: 257-278$
20. Tian Z, Wang J, Wang J, Zhang H (2017) Simplified neutrosophic linguistic multi-criteria group decision-making approach to green product development. Group Decis Negot 26:597-627

21. Nabeeh NA, Abdel-Basset M, El-Ghareeb HA, Aboelfetouh A (2019) Neutrosophic multi-criteria decision making approach for IoT-based enterprises. IEEE Access 7:59559-59574

22. Dubois D, Parade H (1978) Operation on fuzzy number. Int J Fuzzy Syst 9:613-626

23. Kandel A, Byatt WJ (1978) Fuzzy differential equations. In: Proceedings of international conference cybernetics and society, Tokyo, pp 1213-1216

24. Friedmen M, Ming M, Kandel A (1996) Fuzzy derivatives and fuzzy Cauchy problems using LP metric. In: Ruan D (ed) Fuzzy logic foundations and industrial applications. Kluwer Dordrecht, pp 57-72

25. Bede B, Gal SG (2005) Generalizations of the differentiability of fuzzy number-valued functions with applications to fuzzy differential equations. Fuzzy Sets Syst 151:581-599

26. Chalco-Cano Y, Román-Flores H (2008) On new solutions of fuzzy differential equations. Chaos Solitons Fractals 38:112-119

27. Stefanini L, Bede B (2009) Generalized Hukuhara differentiability of interval-valued functions and interval differential equations. Nonlinear Anal 71:1311-1328

28. Bede B, Stefanini L (2013) Generalized differentiability of fuzzyvalued functions. Fuzzy Sets Syst 230:119-141

29. Khastana A, Rodriguez-Lopezb R (2016) On the solutions to first order linear fuzzy differential equations. Fuzzy Sets Syst 295:114 135

30. Mondal SP, Roy TK (2013) First Order linear homogeneous fuzzy ordinary differential equation based on lagrange multiplier method. J Soft Comput Appl 2013:1-17

31. Mahapatra GS, Roy TK (2009) Reliability evaluation using triangular intuitionistic fuzzy numbers arithmetic operations. World Acad Sci Eng Technol 50:574-581

32. Kumar M, Yadav SP, Kumar S (2011) A new approach for analyzing the fuzzy system reliability using intuitionistic fuzzy number. Int $\mathbf{J}$ Ind Syst Eng 8(2):135-156

33. Shaw AK, Roy TK (2013) Trapezoidal Intuitionistic Fuzzy Number with some arithmetic operations and its application on reliability evaluation. Int J Math Oper Res 5(1):55-73

34. Wang J, Nie R, Zhang H, Chen X (2013) New operators on triangular intuitionistic fuzzy numbers and their applications in system fault analysis. Inf Sci 251:79-95

35. Wan S (2013) Power average operators of trapezoidal intuitionistic fuzzy numbers and application to multi-attribute group decision making. Appl Math Model 37:4112-4126

36. Mondal SP, Roy TK (2014) First order homogeneous ordinary differential equation with initial value as triangular intuitionistic fuzzy number. J Uncertain Math Sci 2014:1-17

37. Neutrosophic precalculus and neutrosophic calculus, by Florentin Smarandache, Sitech Educational, EuropaNova, Brussels, Belgium (2015)

38. Sumathi IR, Mohana Priya V (2018) A new perspective on neutrosophic differential equation. Int J Eng Technol 7(4.10):422-425

Publisher's Note Springer Nature remains neutral with regard to jurisdictional claims in published maps and institutional affiliations. 